\title{
Results of Total Knee Arthroplasty with NexGen LPS- flex Implant Using Navigation System (Brain Lab): Results with a 5-year Follow-up
}

Chul Hyung Kang, MD, $\mathrm{PhD}^{1}$, Kyung Jae Lee, $\mathrm{MD}, \mathrm{PhD}^{1}$, Ki Cheor Bae, $\mathrm{MD}, \mathrm{PhD}^{1}$, Chul Hyun Cho, MD, $\mathrm{PhD}^{1}$, Si Wook Lee, $\mathrm{MD}^{1}$, Hong Kwan Shin, $\mathrm{MD}^{2}$, Young Kook Lee, $\mathrm{MD}^{2}$ and Ji Suk Bae, $\mathrm{MD}^{3}$

${ }^{1}$ Department of Orthopedic Surgery, Keimyung University School of Medicine, Daegu; ${ }^{2}$ Department of Orthopedic Surgery, Hanmi Hospital, Daegu; ${ }^{3}$ Department of Preventive Medicine, Catholic University of Daegu School of Medicine, Daegu, Korea

Purpose: To evaluate the clinical and radiological results of patients that underwent total knee arthroplasty (TKA) with a NexGen LPS-Flex implant using a Navigation system (Brain Lab).

Materials and Methods: Between January 2001 and December 2005, 55 knees in 46 patients which used the NexGen LPS-Flex implant with a Navigation system (Brain Lab) for primary TKA were clinically and radiologically evaluated after a minimum follow-up of 5 years. Evaluation included preoperative and postoperative range of motion (ROM), Knee Society Score (KSS), tibio-femoral angle and postoperative complications.

Results: Knee ROM was increased from $118.9^{\circ}$ preoperatively to $126.9^{\circ}$ at the last follow up. In addition, the preoperative flexion contracture improved from $6.5^{\circ}$ to $1.8^{\circ}$ postoperatively. The mean KSS and functional score were improved from 59.8 and 51.2 to postoperative scores of 86.4 and 85.2 respectively. The rate of appearance of radiolucency in X-ray was $21.8 \%$. One case of superficial skin infection and one case of aseptic loosening were noted as complications but, did not require a revision surgery.

Conclusions: TKA with NexGen LPS-Flex implant using Navigation system (Brain Lab) showed satisfactory improvement in pain and function, but more long term follow up will be needed to complete verification.

Key words: Navigation system, NexGen, Total knee arthroplasty.

\section{Introduction}

Total knee arthroplasty (TKA) has been performed for the treatment of severe knee arthritis presenting with pain and limited range of motion (ROM). With improvements in surgical techniques and implant designs, there has been much recent

Received January 24, 2012; Revised (1st) April 12, 2012;

(2nd) July 9, 2012; Accepted July 9, 2012.

Correspondence to: Ki Cheor Bae, MD.

Department of Orthopedic Surgery, Keimyung University School of

Medicine, 56 Dalseong-ro, Jung-gu, Daegu 700-712, Korea.

Tel: +82-53-250-7729, Fax: +82-53-250-7205

Email: bkc@dsmc.or.kr

This is an Open Access article distributed under the terms of the Creative Commons Attribution Non-Commercial License (http://creativecommons.org/licenses/by-nc/3.0/) which permits unrestricted non-commercial use, distribution, and reproduction in any medium, provided the original work is properly cited. advancement in TKA. Factors that determine the success of TKA include proper patient selection, implant design, precise surgical technique, and pre-and postoperative patient management. In particular, the clinical prognosis and long-term longevity of TKA are dependent on proper implant location and precise lower limb alignment ${ }^{1,2)}$. Bathis et al. ${ }^{3)}$ reported that the use of navigation in TKA can be effective in improving the accuracy of the lower limb alignment and implant placement, whereas Jenny and Boeri ${ }^{4)}$ reported that the navigation assistance was not necessarily related to the improved accuracy.

The NexGen (Zimmer Inc., Warsaw, IN, USA) knee implants were designed in mid 1990s and have been evolved to enable hyperflexion. The NexGen LPS-Flex knee implants allow for combination of various implants and greater conformity of articulating surfaces with anatomical femoral component design and improved patellar tracking, interchangeability of different sizes of components, and a variety of surgical equipment selection. Some studies have shown promising results of TKA using the NexGen LPS-Flex knee implants, ${ }^{5,6)}$.

However, mid- or long-term follow-up results of navigation- 
assisted TKA using NexGen LPS-Flex knee implants have not been sufficient to evaluate the hyperflexion design prosthesis. In the present study, we analyzed the clinical results of TKA with the NexGen LPS-Flex implant using a navigation system (Brain Lab, Vector Vision, Heirnstettern, Germany).

\section{Materials and Methods}

\section{Study Population}

Of the patients who underwent TKA with the NexGen LPSFlex knee implant under the diagnosis of primary knee arthritis between January 2001 and December 2005, 46 patients (55 knees) who were available for $\geq 5$ years of clinical and radiological follow-up were included in this study. The surgery was performed using the Brain Lab navigation system (Fig. 1). There were 4 males and 42 females. The mean age of the patients was 67 years and 29 of them were in their $60 \mathrm{~s}$. The indications for surgery were degenerative arthritis in 43 patients (50 knees) and rheumatoid arthritis in 3 patients ( 5 knees). The surgery was bilateral in 9 patients (18 knees) and unilateral in 37 patients (Table 1).

Bone cement was used for femoral and tibial component fixation. A posterior stabilized type (PS type) prosthesis was used in 35 knees and a cruciate retaining type (CR type) prosthesis in 20 knees.

The patella was replaced in 45 knees. Patellar tracking was assessed intraoperatively after implant fixation and determination of polyethylene thickness using the no thumb technique. A lateral release was performed in all knees.

\section{Surgical Technique}

All surgery was performed through the midvastus approach by the same surgeon. The navigation system was used to measure the femorotibial alignment and rotation on the anteroposterior
(AP) and sagittal planes, and assess the lower limb alignment on the AP and sagittal planes and soft tissue balance. After implant insertion, femoral cutting angle (FCA, the angle between the femoral component and the mechanical axis on the AP view), tibia cutting angle (TCA, the angle between the tibial component and the mechanical axis on the AP view), and tibia cutting slope (TCS, posterior tibial slope of the tibial component on the sagittal view) were measured using femoral and tibial cutting block adaptors as verification tools. PS type or CR type NexGen LPSFlex knee prosthesis was implanted.

Postoperatively, cold compression dressing and anti-embolism stockings were used to prevent swelling and deep vein thrombosis. At 2-3 days postoperatively, passive joint exercises were started after removal of the drainage catherter. At 5-7 days postoperatively, physical therapy for weight-bearing and walking was initiated.

\section{Assessments}

For clinical assessment, the knee society score (KSS) and functional score were evaluated preoperatively and at the last follow-up ${ }^{7)}$. For radiological assessment, standing AP and lateral radiographs of the affected limb and standing AP radiographs of both lower extremities taken preoperatively, postoperatively,

Table 1. Demographic Characteristics of Study Participants

\begin{tabular}{lccc}
\hline Characteristic & PS type $(\mathrm{n}=35)$ & CR type $(\mathrm{n}=20)$ & $\mathrm{p}$-value \\
\hline Age $(\mathrm{yr})$ & $67.6 \pm 6.4$ & $65.9 \pm 5.1$ & 0.31 \\
Gender & & & \\
Male & $5(14.3)$ & $0(-)$ & \\
Female & $30(85.7)$ & $20(100.0)$ & \\
\hline
\end{tabular}

Values are presented as mean \pm standard deviation or number (\%). PS: posterior stabilized, CR: cruciate retaining.

${ }^{a)} \mathrm{p}$-values were calculated by independent t-tests or Fisher's exact tests.
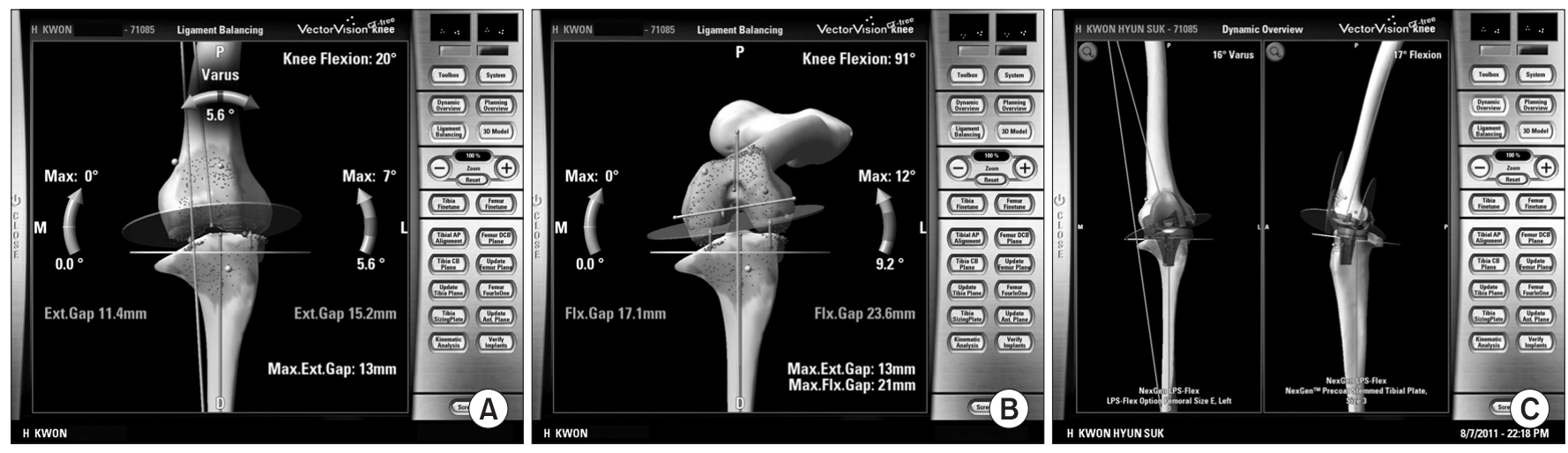

Fig. 1. Soft tissue balancing under navigation guide in primary total knee arthroplasty. Navigation helps to check the balance of extension gap (A), flexion gap (B), and dynamic overview showed the varus deformity and flexion contracture (C). 
and at the last follow-up were evaluated using the knee society radiographic evaluation system. Changes in the femorotibial angle were measured. To assess the implant position, the valgus angle of the femoral component and the varus angle of the tibial component $(\beta)$ were measured on the AP radiographs and the flexion angle of the femoral component $(\gamma)$, and the posterior slope of the tibial component were measured on the lateral radiographs.

The bone-cement or bone-implant contact area of the femoral component was divided into 7 compartments on the lateral radiograph, and that of the tibial component was divided into 7 compartments on the AP radiograph and 3 compartments on the lateral radiograph. The width of a radiolucent line in each compartment was marked in units of $0.5 \mathrm{~mm}$. The presence of a radiolucent line was considered insignificant if the sum of the recorded values was $\leq 4$, a constant follow-up was considered required if it was between 5 and 9 , and fixation failure was considered highly probable if it was $\geq 10$ regardless of the presence of clinical symptoms ${ }^{8}$. If a change in implant position or severe displacement is observed, fixation was considered to have failed regardless of the presence of a radiolucent line.

\section{Statistical Analysis}

SPSS ver. 14.0 (SPSS Inc., Chicago, IL, USA) was used for statistical analysis. Independent t-test and Pearson chi-square test were used for intergroup comparisons. The level of confidence was set at $95 \%$. The independent $t$-test was used for comparisons between the PS and CR groups whereas the Fisher's exact test was used for comparisons of the frequency of the appearance of radiolucent lines. Pre- and postoperative clinical results were compared between the groups using a paired t-test. A p-value $<0.05$ by a two-sided test was considered statistically significant.

\section{Results}

\section{Clinical Results}

The mean ROM was increased significantly from $118.9^{\circ}$ (range,

Table 2. Clinical Results

\begin{tabular}{lccc}
\hline \multicolumn{1}{c}{ Variable } & Preoperative & $\begin{array}{c}\text { Immediate } \\
\text { postoperative }\end{array}$ & p-value \\
\hline Mechanical axis & $-12.5 \pm 5.8$ & $1.2 \pm 1.6$ & 0.028 \\
Flexion contracture & $6.5 \pm 5.8$ & $1.8 \pm 3.9$ & 0.014 \\
Range of motion & $118.9 \pm 15.8$ & $126.9 \pm 12.3$ & 0.012 \\
Knee Society Score & $59.9 \pm 3.7$ & $86.4 \pm 3.7$ & 0.005 \\
Functional Score & $51.2 \pm 4.3$ & $85.2 \pm 2.8$ & 0.007 \\
\hline
\end{tabular}

$90^{\circ}-150^{\circ}$ ) preoperatively to $126.9^{\circ}$ (range, $100^{\circ}-150^{\circ}$ ) at the last follow-up $(\mathrm{p}<0.05)$. The mean flexion contracture was improved from $6.5^{\circ}$ (range, $0^{\circ}-25^{\circ}$ ) preoperatively to $1.8^{\circ}$ (range, $0^{\circ}-10^{\circ}$ ) postoperatively.

A statistically significant improvement was found in the mean KSS from 59.8 points preoperatively to 86.3 at the last followup $(\mathrm{p}<0.05)$, and in the mean function score from 51.2 points preoperatively to 85.2 points at the last follow-up (Table 2).

A PS type prosthesis was used in 35 knees where severe flexion contracture or varus/valgus deformity was present, normal knee function appeared unattainable with the use of a CR type prosthesis due to severe posterior cruciate ligament (PCL) injury, or severe asymmetrical extension-flexion gap was observed. However, no statistically significant differences were found between the PS group and the CR group (Table 3).

\section{Radiological Results}

The mean mechanical axis that extends from the center of the femoral head through the centers of the femoral condyle and tibial condyle to the center of the ankle on the standing AP radiograph was $12.5^{\circ}$ varus $\left(24^{\circ}\right.$ varus to $5^{\circ}$ valgus) preoperatively and $1.2^{\circ}$ varus ( $8^{\circ}$ varus to $1^{\circ}$ valgus) at the last follow-up. The mean femorotibial angle was $5.2^{\circ}$ valgus ( $\left(1^{\circ}\right.$ varus to $10^{\circ}$ valgus). Regarding the implant position, the mean valgus angle of the femoral component $(\alpha)$ was $95.2^{\circ}$ (range, $91^{\circ}-99^{\circ}$ ) and the mean varus angle of the tibial component $(\beta)$ was $92.3^{\circ}$ (range, $89^{\circ}-97^{\circ}$ )

Table 3. Means and Standard Deviations for Clinical Results Pre- and Postoperation by Treatment

\begin{tabular}{|c|c|c|c|}
\hline Variable & $\begin{array}{l}\text { Posterior stabilized } \\
\qquad(\mathrm{n}=35)\end{array}$ & $\begin{array}{l}\text { Cruciate retaining } \\
\qquad(\mathrm{n}=20)\end{array}$ & p-value ${ }^{a)}$ \\
\hline \multicolumn{4}{|l|}{ Range of motion } \\
\hline Preoperative & $121.1 \pm 15.9$ & $115.0 \pm 15.4$ & 0.17 \\
\hline Postoperative & $129.4 \pm 11.9$ & $122.5 \pm 12.1$ & 0.08 \\
\hline p-values ${ }^{\text {b) }}$ & 0.003 & 0.015 & \\
\hline \multicolumn{4}{|l|}{ Knee Society Score } \\
\hline Preoperative & $59.6 \pm 3.7$ & $60.3 \pm 3.8$ & 0.51 \\
\hline Postoperative & $86.8 \pm 3.7$ & $85.7 \pm 3.6$ & 0.28 \\
\hline p-values ${ }^{\text {b) }}$ & 0.003 & 0.006 & \\
\hline \multicolumn{4}{|l|}{ Functional Score } \\
\hline Preoperative & $51.0 \pm 4.4$ & $51.6 \pm 4.0$ & 0.63 \\
\hline Postoperative & $85.4 \pm 2.8$ & $84.9 \pm 3.0$ & 0.53 \\
\hline p-values ${ }^{\text {b) }}$ & 0.008 & 0.005 & \\
\hline
\end{tabular}


Table 4. Radiologic Results

\begin{tabular}{lccc}
\hline Variable & Immediate postoperative & 5 years follow-up & p-value \\
\hline$\alpha$ angle & $95.2 \pm 2.2$ & $95.6 \pm 2.3$ & 0.0003 \\
$\beta$ angle & $92.3 \pm 1.9$ & $91.7 \pm 1.9$ & 0.0012 \\
$\gamma$ angle & $3.2 \pm 1.4$ & $3.2 \pm 1.6$ & 0.0001 \\
$\delta$ angle & $84.7 \pm 1.9$ & $84.5 \pm 2.4$ & 0.0001 \\
\hline
\end{tabular}

on the postoperative AP radiographs. The mean flexion angle of the femoral component $(\gamma)$ was $3.24^{\circ}$ (range, $0^{\circ}-6^{\circ}$ ) and the mean posterior slope of the tibial component $(\delta)$ was $84.7^{\circ}$ (range, $81^{\circ}-89^{\circ}$ ) on the lateral radiographs. The mean valgus angle of the femoral component $(\alpha)$ was $95.6^{\circ}$ (range, $93^{\circ}-100^{\circ}$ ) at the last follow-up. The mean varus angle of the tibial component $(\beta)$ was $92^{\circ}$ (range, $87^{\circ}-95^{\circ}$ ), the mean flexion angle of the femoral component $(\gamma)$ was $3.2^{\circ}$ (range, $0^{\circ}-8^{\circ}$ ), and the mean posterior tibial slope of the tibial component $(\delta)$ was $85^{\circ}$ (range, $79^{\circ}-90^{\circ}$ ) at the last follow-up. There was no statistically significant change between the postoperative and the last follow-up examinations ( $p>0.05$ ). There was no $\geq 10^{\circ}$ difference between the postoperative and last follow-up values, and accordingly, the component position and alignment were considered well-maintained (Table 4).

Radiolucent lines were observed in 12 knees $(21.8 \%)$ at the last follow-up and the mean sum of the values was 0.3 points. Regarding the frequency of the appearance of radiolucent lines, a radiolucent line was observed anterior to the femoral component in 6 knees, posterior to the femoral component in 4 knees, medial to the tibial component in 2 knees, and anterior to the tibial component in 2 knees. There was no significant relationship between the presence of a radiolucent line and clinical results ( $>0.05$ ).

There was 1 case of skin infection and 1 case of aseptic loosening postoperatively. The skin infection was observed in a patient who had been medicated for diabetes and hypertension and healed with wound dressing in 3 weeks. In the patient with aseptic loosening, minor sink down of the tibial component was observed. The patient has neither presented with pain nor symptoms in the follow-up examinations that have been conducted with an interval of 5 months. Reoperation was not performed in any cases.

\section{Discussion}

As part of an effort to improve the accuracy of TKA, navigationor computer-assisted TKA techniques have been introduced recently. Some studies have shown that the recent techniques are more effective for precise realignment of the mechanical axis and implant positioning ${ }^{1,3,4)}$.

Stulberg ${ }^{9)}$ assessed implant position and lower limb alignment using a navigation system after conventional TKA. The conventional mechanical instrumentation system had a tendency to leave the femoral component in varus, flexion, and internal rotation and the tibial component in varus after navitagionassisted TKAs. They explained that this tendency occurs when the femoral intramedullary alignment rod is short or narrow, compared to the medullary cavity or inserted medial to the center of the medullary cavity in the presence of severe deformity of the femur or tibia. Hart et al. ${ }^{10)}$ compared the radiological results of navigation assisted TKAs and conventional TKAs. Although there was no significant intergroup difference, the number of cases with $\geq 2^{\circ}$ deviation of the femoral or tibial component from the ideal value was significantly lower after navigation-assisted TKAs. Sparmann et al. ${ }^{11)}$ reported that the use of a navigation system improved the accuracy of TKA by maintaining $<1^{\circ}$ error for each stage of the procedure reducing the malalignment error. In the current study, we found $\geq 3^{\circ}$ of malalignment in 9 knees (16.3\%).

Hart et al. ${ }^{10)}$ recommended navigation-assisted TKAs to inexperienced surgeons and even to skilled surgeons for the purpose of reducing the margin of error, even if the use of a navigation system would not make a difference in the results.

The NexGen LPS-Flex knee implant (Zimmer Inc.) was designed to have more bumps on the medial and lateral condyles of the femoral component for advanced stability, and a more posteriorly and proximally positioned femoral cam to minimize the risk of dislocation during knee flexion compared to the previous model ${ }^{5,6)}$.

Bertin $^{12)}$ followed 251 knees for 5-7 years after TKA using an NexGen posterior cruciate-retaining LPS-Flex implant and the mean ROM was $123^{\circ}$, which is similar to $126.9^{\circ}$ obtained in our patients at the last follow-up.

PCL preservation in TKA has been controversial. The advantages of PCL preservation include enhanced knee flexion through greater femoral rollback, increased resistance to posterior shear force for stability, and preservation of the joint surface height and proprioception. On the other hand, PCLretaining TKA is difficult to perform in knees with severe deformity or bone defects, and has been associated with joint instability and early polyethylene wear ${ }^{13,14)}$. PCL-substituting TKA is easy to perform with minimal tibial resection, facilitates deformity correction, and reduces the risk of polyethylene 


\section{Kang et al. Results of TKA with NexGen LPS-flex Implant Using Navigation System}

wear by maximizing the contact area ${ }^{5,15)}$. Jung et al. ${ }^{6)}$ reported that the clinical and radiological scores were significantly low in patients with severe flexion contracture or deformity after PCL-substituting TKA. However, we could not find statistically significant differences in radiological results between the PCLsubstituting group (PS group) and PCL-retaining group (CR group) (Table 3).

There is disagreement among authors on the patella resurfacing. Keblish et al ${ }^{16}{ }^{16}$ reported that patellar resurfacing did not result in significant intergroup difference after bilateral TKA. On the other hand, Scott and Reilly ${ }^{17)}$ recommended to resurface the patella in knees with inflammatory disorders, including rheumatoid arthritis, whereas to consider several factors including the shape of the patella and cartilage condition in knees with degenerative arthritis. In our study, we selectively performed patella resurfacing on 45 knees by taking the patellar cartilage condition and movement of the femoral trochlea into consideration.

The importance of femorotibial alignment after TKA has been documented in many studies. Lotke and Ecker ${ }^{18)}$ reported that $3^{\circ}$ to $7^{\circ}$ of valgus after TKA is the most ideal alignment. Considering that the mean postoperative femorotibial angle was $5.2^{\circ}$ of valgus in our study, deformity correction was satisfactory in our patients.

Kraay et al. ${ }^{19)}$ reported that periprosthetic radiolucent lines were most frequently observed under the proximal portion of anterior flange of the femoral component. The radiolucency was attributed to initial incongruities between the cut surface of the anterior femur and the implant, and slight flexion of the implant that resulted in increased stress. King and Scott ${ }^{20)}$ observed radiographic evidence of femoral component loosening in 15 out of 1,600 knees after TKA, 13 of which occurred in zone 4 and a radiolucent line was noted immediately after surgery in 8 knees. They attributed early loosening to improper resection of the posterior condyle, poor cementing technique, and inadequate support of the prosthetic posterior condyle due to deficient bone stock. In our study, radiolucent lines were more common around the femoral component ( 9 cases) than the tibial component (4 cases). The presence of radiolucency was seen in zones 2 and 5 around the femur (anterior femur on the lateral view), in medial zones 1 and 2 (on the AP view) and zone 1 (on the lateral view) around the tibia, which are similar to the results of Kraay et al. ${ }^{19)}$ except for the lower frequency.

In 12 knees (21.8\%) with radiolucency, substantial implant migration or loosening was not noted. In 1 knee with severe bone defect in the posteromedial aspect of the tibia and severe varus deformity, allogeneic femoral head bone graft was fixated using screws in the defect and TKA was performed using a PCLsubstituting implant.

In this study, we did not make direct comparisons with the conventional TKA, but the clinical improvement after TKA with NexGen LPS-Flex implant using a navigation system was relatively similar to that of other studies using NexGen implants ${ }^{4,14}$. The frequency of the appearance of radiolucent lines was remarkably lower in our study compared to those studies. We attributed this to the use of a navigation system that enabled precise posterior bone resection. However, we think a long-term follow-up study should be performed to validate the analysis. Bae et al. ${ }^{1)}$ reported excellent radiological results of navigationassisted TKA, but suggested that the clinical influence of the use of a navigation system should be assessed in a long-term followup study.

\section{Conclusions}

TKA with NexGen LPS-Flex implant using a navigation system resulted in good ROM and functional recovery, low complication rates, and satisfactory radiological outcomes at $\geq 5$ years after surgery. However, we think periprosthetic radiolucency and component loosening should be assessed in further studies involving a larger patient population and a longer follow-up period.

\section{References}

1. Bae DK, Yoon KH, Song SJ, Kim SG, Park KJ. Intraoperative versus postoperative measurement in total knee arthroplasty using computer-assisted orthopaedic surgery: accuracy of CAOS. J Korean Orthop Assoc. 2005;40:168-73.

2. Knutson K, Lindstrand A, Lidgren L. Survival of knee arthroplasties. A nation-wide multicentre investigation of 8000 cases. J Bone Joint Surg Br. 1986;68:795-803.

3. Bathis H, Perlick L, Tingart M, Luring C, Zurakowski D, Grifka J. Alignment in total knee arthroplasty. A comparison of computer-assisted surgery with the conventional technique. J Bone Joint Surg Br. 1998;86:682-8.

4. Jenny JY, Boeri C. Computer-assisted implantation of a total knee arthroplasty: a case-controlled study in comparison with classical instrumentation. Rev Chir Orthop Reparatrice Appar Mot. 2001;87:645-52.

5. Choi CH, Kim JH, Cho YJ, Cho JH, Chung HK. Results of total knee arthroplasty with NexGen(R) system (5-year follow up). J Korean Orthop Assoc. 2003;38:336-41. 
6. Jung KH, Cho SD, Go SH, Cha JR, Lee CC, Youm YS, Park KB. Total knee arthroplasty with NexGen(R) system: 3-8 year follow-up results. J Korean Orthop Assoc. 2007;42:7306.

7. Insall JN, Scott WN. Surgery of the knee. Vol. 2. 3rd ed. New York: Churchill Livingstone; 2001. p1663-4.

8. Ewald FC. The Knee Society total knee arthroplasty roentgenographic evaluation and scoring system. Clin Orthop Relat Res. 1989;(248):9-12.

9. Stulberg SD. How accurate is current TKR instrumentation? Clin Orthop Relat Res. 2003;(416):177-84.

10. Hart R, Janecek M, Chaker A, Bucek P. Total knee arthroplasty implanted with and without kinematic navigation. Int Orthop. 2003;27:366-9.

11. Sparmann M, Wolke B, Czupalla H, Banzer D, Zink A. Positioning of total knee arthroplasty with and without navigation support. A prospective, randomised study. J Bone Joint Surg Br. 2003;85:830-5.

12. Bertin KC. Cruciate-retaining total knee arthroplasty at 5 to 7 years followup. Clin Orthop Relat Res. 2005;(436):177-83.

13. Insall JN, Lachiewicz PF, Burstein AH. The posterior stabilized condylar prosthesis: a modification of the total condylar design. Two to four-year clinical experience. J Bone
Joint Surg Am. 1982;64:1317-23.

14. Scott WN, Rubinstein M, Scuderi G. Results after knee replacement with a posterior cruciate-substituting prosthesis. J Bone Joint Surg Am. 1988;70:1163-73.

15. Freeman MA, Railton GT. Should the posterior cruciate ligament be retained or resected in condylar nonmeniscal knee arthroplasty? The case for resection. J Arthroplasty. 1988;3 Suppl:S3-12.

16. Keblish PA, Varma AK, Greenwald AS. Patellar resurfacing or retention in total knee arthroplasty. A prospective study of patients with bilateral replacements. J Bone Joint Surg Br. 1994;76:930-7.

17. Scott RD, Reilly DT. Pros and cons of patellar resurfacing in total knee replacement. Orthop Trans. 1980;4:328-9.

18. Lotke PA, Ecker ML. Influence of positioning of prosthesis in total knee replacement. J Bone Joint Surg Am. 1977;59:779.

19. Kraay MJ, Meyers SA, Goldberg VM, Figgie HE 3rd, Conroy PA. "Hybrid" total knee arthroplasty with the Miller-Galante prosthesis. A prospective clinical and roentgenographic evaluation. Clin Orthop Relat Res. 1991;(273):32-41.

20. King TV, Scott RD. Femoral component loosening in total knee arthroplasty. Clin Orthop Relat Res. 1985;(194):285-90. 